Hogan, N. J., Urban, A. S., Ayala-Orozco, C., Pimpinelli, A., Nordlander, P., \& Halas, N. J. (2014). Nanoparticles Heat through Light Localization. Nano Letters. doi:10.1021/n15016975

\title{
Nanoparticles Heat through Light Localization
}

\footnotetext{
${ }^{1}$ Department of Physics and Astronomy

${ }^{2}$ Laboratory for Nanophotonics

${ }^{3}$ Department of Electrical and Computer Engineering

${ }^{4}$ Rice Quantum Institute

${ }^{5}$ Department of Chemistry
}

Rice University, Houston, Texas 77005, United States 


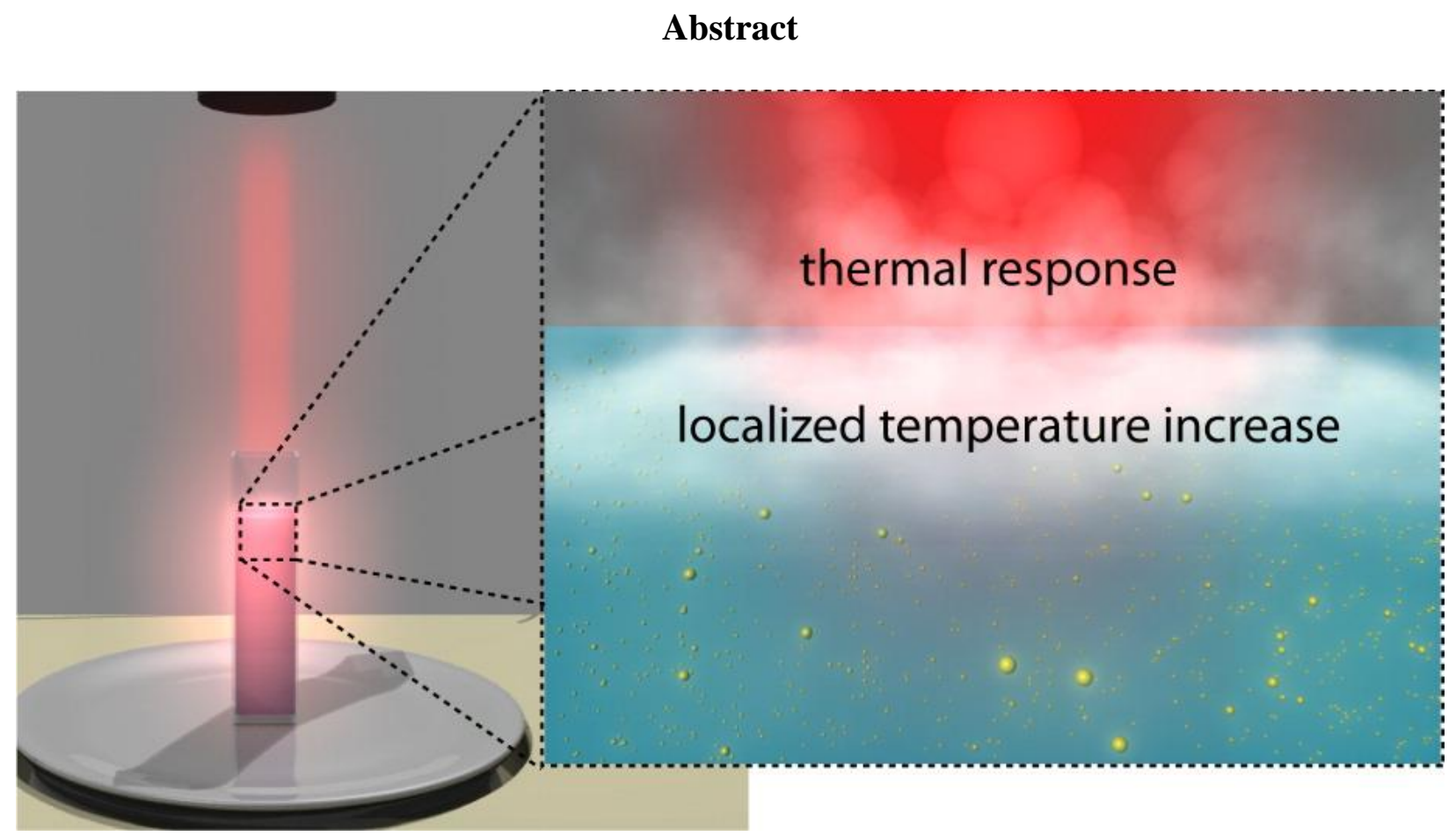

Aqueous solutions containing light-absorbing nanoparticles have recently been shown to produce steam at high efficiencies upon solar illumination, even when the temperature of the bulk fluid volume remains far below its boiling point. Here we show that this phenomenon is due to a collective effect mediated by multiple light scattering from the dispersed nanoparticles.

Randomly positioned nanoparticles that both scatter and absorb light are able to concentrate light energy into mesoscale volumes near the illuminated surface of the liquid. The resulting light absorption creates intense localized heating and efficient vaporization of the surrounding liquid. Light trapping-induced localized heating provides the mechanism for low-temperature lightinduced steam generation and is consistent with classical heat transfer.

Keywords: Plasmon; nanoscale heating; radiative transport 
In a world of ever-increasing energy demand, the harvesting of sunlight has the potential to provide a useful energy source for much of the planet. In addition to the solar energy harvesting technologies currently under development,(1) such as photovoltaics(2) and solar thermal electricity generation,(3) there is avid interest in novel effects that may lend themselves to entirely new approaches. Recently it was reported that light-absorbing nanoparticles, immersed in water and illuminated by sunlight, are capable of generating steam without the necessity of heating the entire fluid volume, resulting in remarkably high steam generation efficiencies. It was initially observed that over $80 \%$ of the absorbed energy was utilized for steam generation.(4) Because of the large interparticle distances of ten microns or more in these solutions, the particles were assumed to be both optically and thermally isolated. A phase change localized around the individual particles, an effect well-established at higher illumination levels,(5) was proposed as a possible explanation for this process. However, it has been noted that at such illumination intensities a temperature increase at the nanoparticle surface commensurate with vapor nucleation is not in agreement with other single particle measurements and would require nonclassical effects.(6) It has been suggested that collective thermal effects are important in explaining the response of such systems.(7-11) Here we show, however, that collective thermal effects alone are not sufficient to explain the thermal response of these systems. Rather, light trapping by solutions of particles that simultaneously absorb and scatter light results in highly localized heating, an unanticipated effect that, when combined with classical heat transfer, provides an accurate theoretical description of the system.

When light interacts with an ensemble of randomly dispersed particles, as in a fluid environment, the light can be either scattered or absorbed, or both, depending on the properties of the particles. When the average distance between particles in a solution is smaller than the wavelength of light, the wave nature of light must be explicitly considered, and multiple scattering events can lead to phenomena such as weak localization or Anderson localization of light.(12-16) For the relatively low nanoparticle concentrations used in this study, the wave nature of light is only implicitly used to determine the optical properties of a single particle. Each photon is considered independently in a ballistic transport formulation. Conservation of energy leads directly to an equation of transfer, which in the absence of emission terms is

$$
\frac{1}{c} \frac{\partial I}{\partial t}+\hat{\Omega} \cdot \nabla I+\left(k_{\mathrm{s}}+k_{\mathrm{a}}\right) I=\frac{1}{4 \pi} k_{\mathrm{s}} \int_{4, \pi} p\left(\hat{\Omega}, \hat{\Omega}^{\prime}\right) I \mathrm{~d} \Omega
$$

where $I$ is the specific intensity, $c$ is the speed of light, $\Omega$ is the solid angle, $k_{\mathrm{s}}$ and $k_{\mathrm{a}}$ are the scattering and absorption coefficients, respectively, and $p$ is the phase function of scattered radiation. $(17,18)$ The first two terms on the left-hand side stem from a continuity equation that corresponds to the conservation of photons. The third term represents the exponential attenuation of intensity due to scattering and absorption in the medium and, together with the previous two terms, constitutes the Beer-Lambert (BL) law. The BL law provides a good approximation of light transfer in systems where absorption is much stronger than scattering. The right-hand side represents the contribution to the intensity from photons either scattered prior to absorption or scattered out of the system (Figure 1A). With this term taken into account, approximations are required for the equation to be solvable analytically. When scattering is much stronger than absorption, it reduces to a diffusion equation. $(17,19)$ However, neither the strongly absorbing 
nor strongly scattering approximations apply when particles scatter and absorb with comparable probabilities, and the full equation must be solved. This more general case has been of particular interest for applications in biomedical imaging and for unconventional therapeutics, such as photothermal ablation of tumors.(20-24) To numerically solve this for the case of absorberscatterers, we simulate the fate of each incident photon passing through a random ensemble of particles. $(25,26)$ In the limit of pure absorbers or pure scatterers this approach agrees with both the Beer-Lambert law and the diffusion approximation, respectively (Figure S1, Supporting Information).

A

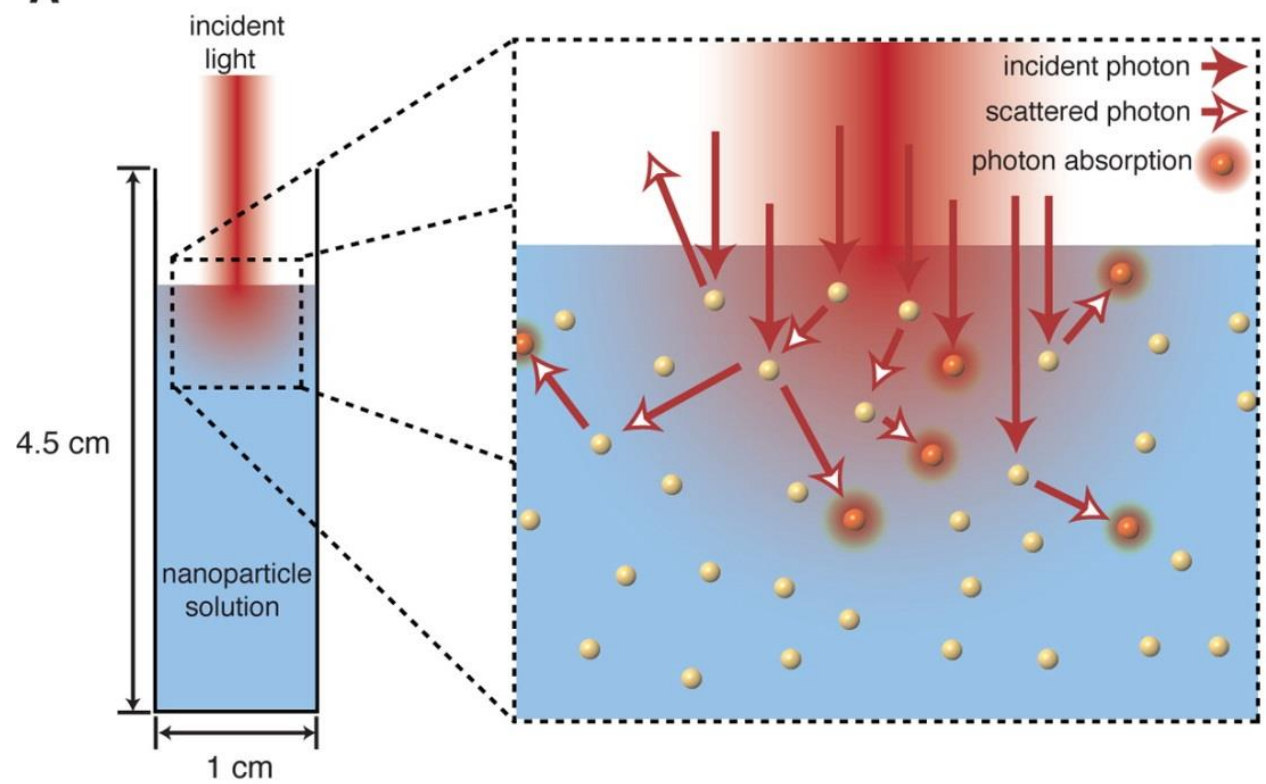

B

Experiment

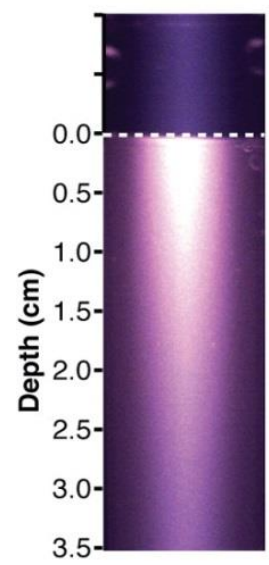

$5 \times 10^{8}$

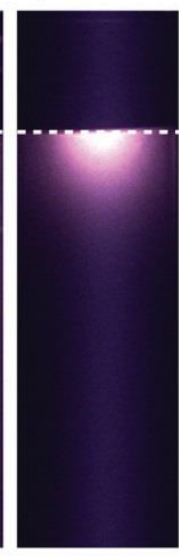

$5 \times 10^{9}$ [nanoparticles $/ \mathrm{ml}$ ]
Simulation

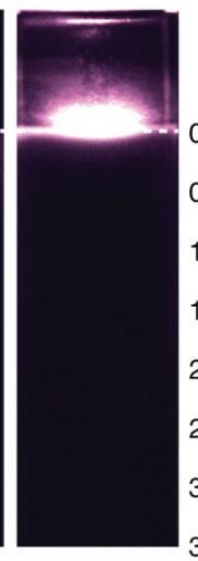

$5 \times 10^{10}$

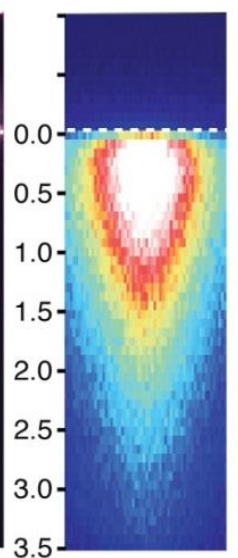

$5 \times 10^{8}$

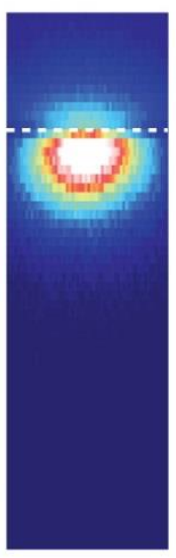

$5 \times 10^{9}$
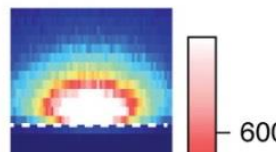

600

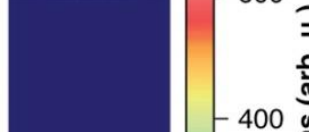

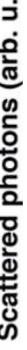

[nanoparticles/ml]

Figure 1. (A) Schematic illustrating characteristic experiment (left) where a dense solution of nanoparticles contained in a cuvette is illuminated with $808 \mathrm{~nm}$ laser light; multiparticle optical interactions in such nanofluids 
(right) where photons are scattered and/or absorbed. (B) Experimentally obtained (left) and Monte Carlo (MC) simulated (right) scattered light as viewed from the side of cuvettes containing nanoshell solutions of the indicated concentrations. Integration times are not the same for all three experiments.

To study light propagation through a solution of nanoparticles that both scatter and absorb light, we fabricated solutions of two different metallo-dielectric nanoparticles: nanoshells and nanomatryoshkas.(27-29) Each type of particle has the same absorption cross section of $\sim 1 \times$ $10^{-10} \mathrm{~cm}^{2}$ for resonant excitation at $808 \mathrm{~nm}$ but substantially different scattering cross sections of $1 \times 10^{-9}$ and $6 \times 10^{-11} \mathrm{~cm}^{2}$, respectively (determined by comparing Mie theory for the two particle geometries with experimentally obtained extinction spectra; Figure S2 A,B, Supporting Information). Nanoshells are primarily scattering particles with a ratio of scattering to absorption efficiency of 10, while nanomatryoshkas are predominantly absorptive, with a scattering to absorption efficiency of 0.6. These particles not only offer tunability of the scattering and absorption efficiencies but also have well-known scattering phase functions,(30-32) a feature not accessible in biological systems. We do not consider nonmetallic absorbers such as carbon nanoparticles in this study due to the inability to accurately measure or control the cross sections. The ability to control all of the experimental parameters allows us to compare experimental results with theoretical predictions rigorously.

First we examine the light-scattering properties of solutions of nanoshell particles, illuminated from above with resonant light at $808 \mathrm{~nm}$ wavelength, as a function of particle concentration (Figure 1B). The experimental images of the light scattered through the side face of the cuvette reveal a decreasing amount of light as the incident beam propagates into the solution. For higher particle concentrations the light does not penetrate as deeply, and strongly increased backscattering off the fluid surface at the highest concentration is apparent. The observed scattered light distributions closely resemble those predicted directly from the multiplescattering simulations (Figure 1B), which incorporate the dipole scattering distribution of the resonant particles.

On the basis of this approach, we examine the effect of light scattering on the fraction of light absorbed by particle solutions over a broad range of concentrations (Figure 2). Here we compare the fractional light absorption, $\eta$, as predicted from a single-scattering corrected BL law calculation(33) with the results of the multiple-scattering simulations (Figure 2A). Multiple scattering begins to affect the fractional light absorption dramatically for nanoshells and nanomatryoshkas at concentrations of $\sim 1 \times 10^{9}$ and $\sim 1 \times 10^{10} \mathrm{NP} / \mathrm{milliliter}(\mathrm{ml})$, respectively. The Beer-Lambert law estimates that $\eta$ saturates at $\sim 0.18$ for nanoshells; when multiple scattering is accounted for, this value is $\sim 0.70$. For nanomatryoshkas, $\eta$ saturates at $\sim 0.60$ in the single-scattering regime but is $>0.90$ when multiple scattering events are taken into account. These very large discrepancies result from the inherent assumption in the Beer-Lambert law that all scattered photons are lost from the beam as it is transmitted through a solution of particles. For absorber-scatterers, this approximation is justified only at low particle concentrations. Multiple scattering events increase the average path length of the photons, which increases the average absorption probability. When the scattering length is comparable to any of the linear dimensions of the solution, a scattered photon is likely to traverse the entire length of the solution without any subsequent interactions. Therefore, at low concentrations (or small volumes), the Beer-Lambert law and the multiple-scattering simulations should agree (Figure 2A (inset)). The concentrations for which the multiple scattering regime begins to deviate strongly 
from the Beer-Lambert law correspond to a scattering length of about $0.5 \mathrm{~cm}$, or half the $1 \mathrm{~cm}$ width of the fluid volume. The distribution of scattering events a photon experiences prior to absorption in each case illustrates this transition quantitatively (Figure 2B).

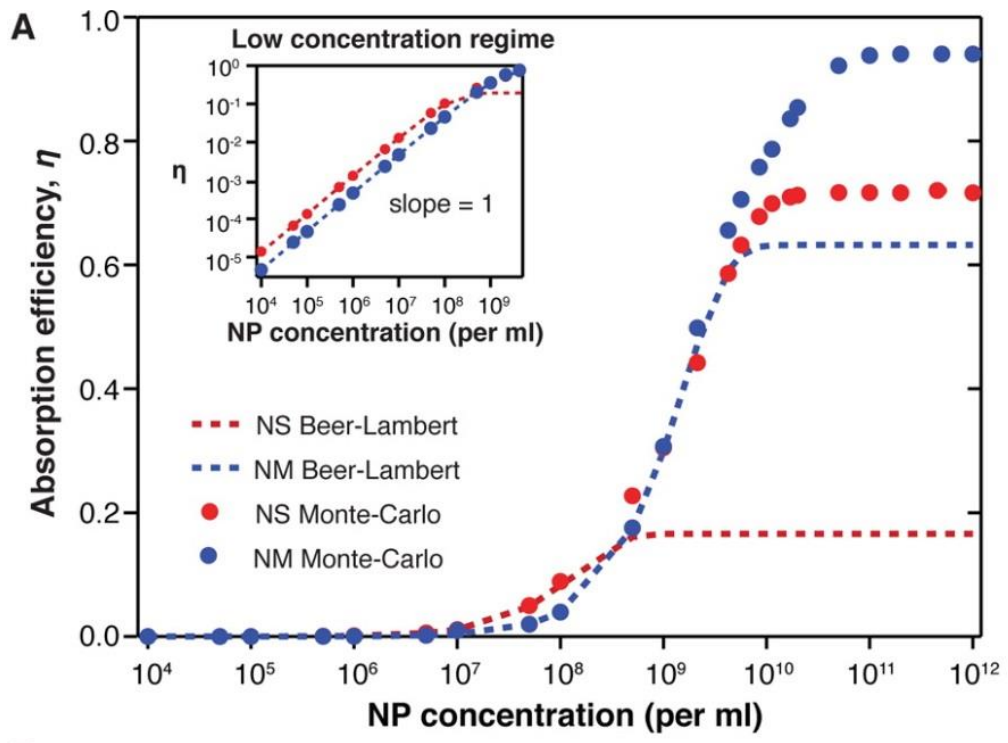

B
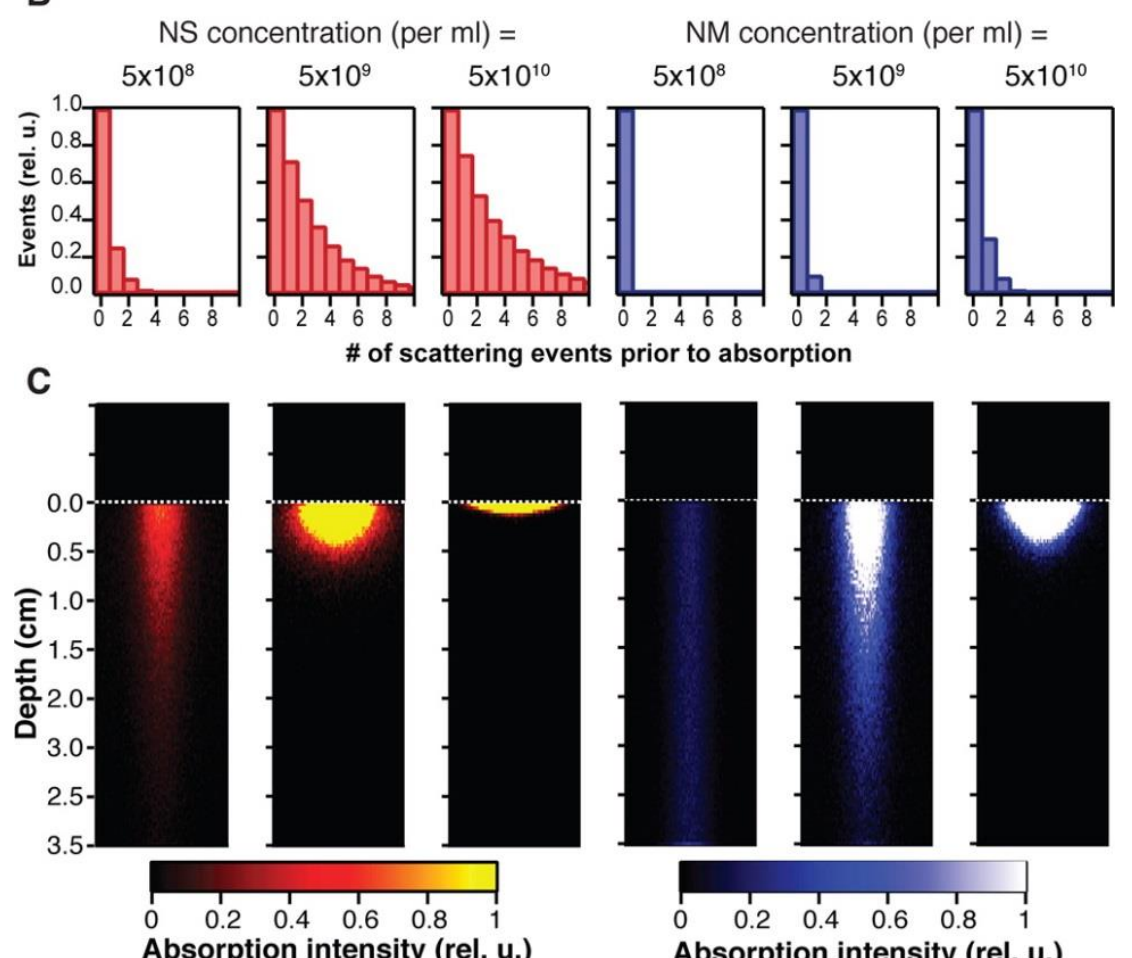

Absorption intensity (rel. u.)

Figure 2. (A) Fraction of incident light absorbed, $\eta$, by a $3.5 \times 1 \times 1 \mathrm{~cm}^{3}$ nanofluid of nanoshells (NS) and nanomatryoshkas (NM) as a function of nanoparticle (NP) concentration (log scale), shown for the single-scattering corrected Beer-Lambert (BL) law and the Monte Carlo (MC) method. Inset: Similar plot except for low concentrations and on a log-log scale to illustrate proportional dependence. (B) Distributions of a number of scattering events prior to absorption of photons in a MC simulation of NS (red, left) and NM (blue, right) solutions of three concentrations. (C) Results of MC simulation of absorption intensity of photons corresponding to concentrations in (B) of NS (red) and NM (blue) solutions. Vertical axis plots penetration depth of absorbed radiation, and horizontal axis represents width of nanofluid. 
Multiple scattering events control the spatial distribution of absorbed photons (Figure $2 \mathrm{C}$ ), which in turn directly determines the thermal response. The distribution of absorbed power defines a heat source density, $q\left(\mathrm{~W} / \mathrm{cm}^{3}\right)$, in a classical heat transfer analysis. We calculate $q$ from

$$
q(x, y, z)=\frac{\eta N_{\mathrm{abs}}(x, y, z) P_{\text {inc }}}{\int N_{\mathrm{abs}}(x, y, z) \mathrm{d} V}
$$

where $\eta$ is the fractional light absorption, $N_{\text {abs }}$ is the distribution of absorbed photons obtained from the multiple-scattering simulations, and $P_{\text {inc }}$ is the incident laser power. A comparison of the localized region of absorbed energy due to multiple scattering by nanoshells or nanomatryoshkas of equal concentrations (Figure 2C) reveals that nanoshell solutions, as primarily scatterers, are able to localize the absorption of light into much smaller volumes than nanomatryoshka solutions. Quantitatively, at the highest concentrations shown in Figure 2C, the exponential decay length of the absorption is a factor of 10 smaller for nanoshells $(\sim 500 \mu \mathrm{m})$ than for nanomatryoshkas $(5 \mathrm{~mm})$. While nanoshells only absorb $70 \%$ of the light at this concentration compared to $94 \%$ for nanomatryoshkas, the maximum of the heat source density, $q$, is almost an order of magnitude larger for nanoshells due to the much smaller absorption volume.

This highly compact heat source density directly dictates the temperature distribution and steam production of the nanoparticle solutions (Figure 3). The experimental geometry was modeled using a 3-dimensional thermal transport finite-element method (FEM, Comsol), incorporating the heat source density obtained from the multiple-scattering simulations for the respective particle type, with no free parameters. First, the temperature evolution of the system, measured at the top and bottom of the nanoparticle solution for six concentrations (Figure 3A) is now accurately described by classical heat transfer with the light trapping-induced heat source density as input. These simulations assume the laser is incident from the top of the solution (Figure S3, Supporting Information), and therefore, the temperature gradient is such that convective forces are negligible. The absence of convective forces reduces the problem of heat conduction. In a comparison between nanoshells and nanomatryoshkas, the temperature increase at the top of the nanoshell solutions is $>10^{\circ} \mathrm{C}$ larger than in nanomatryoshka solutions of equal concentration. This is a direct result of the increased light trapping and localization for nanoshell solutions, which provides an order of magnitude larger maximum heat source density than for nanomatryoshka solutions. The temperature gradient along the axis of light propagation is significantly different between nanoshell and nanomatryoshka solutions, where in the latter case near equivalent temperatures throughout the solution occur at the lowest particle concentrations. 

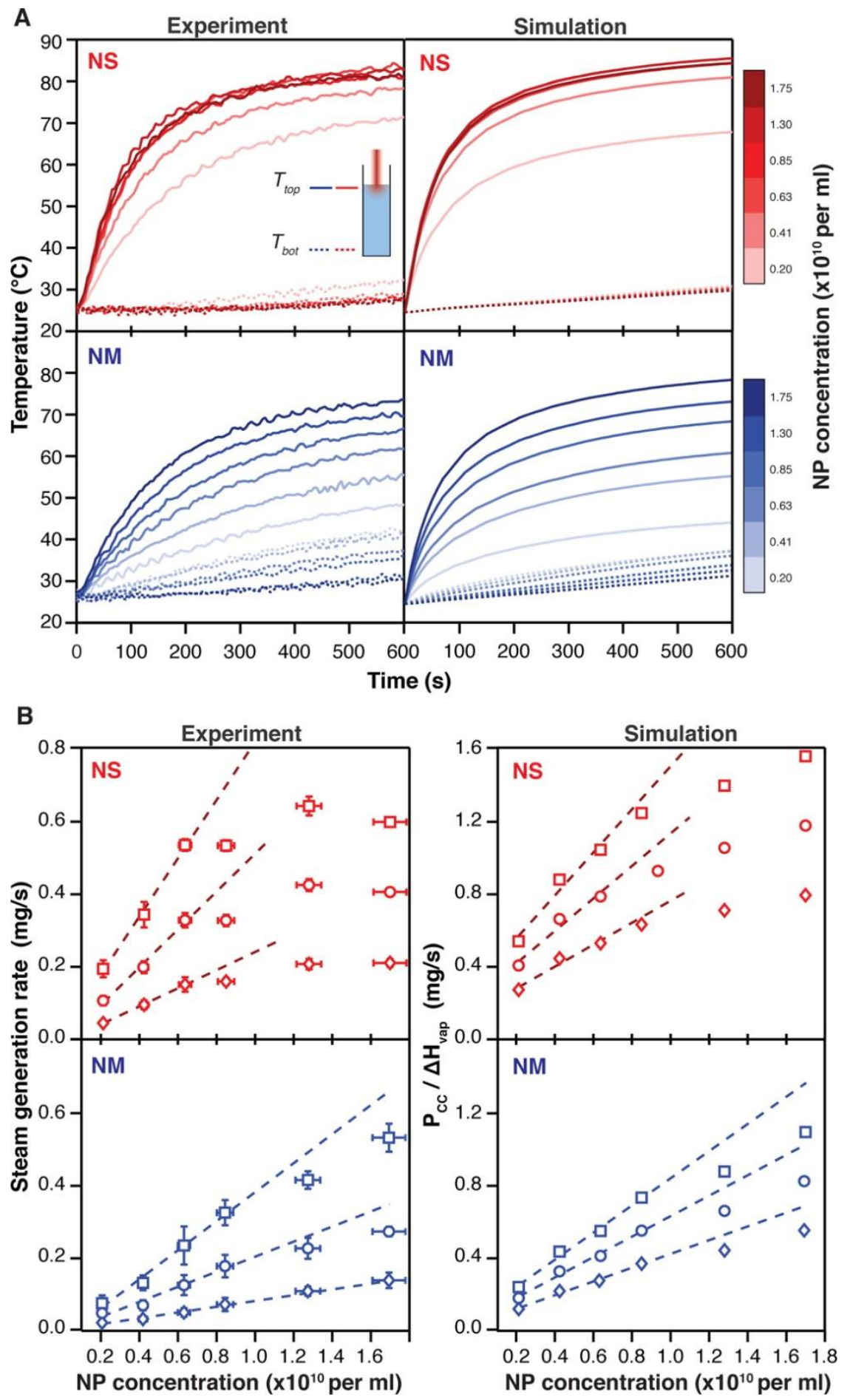

Figure 3. Thermal response of illuminated nanoparticle solutions. (A) Inset shows definition of displayed parameters: temperatures measured at the top and bottom of the solutions are shown as solid and dashed lines, respectively. Experimental and simulated temperature evolution at the top and of illuminated NS (red) and NM (blue) solutions of six different concentrations (color gradient shows increasing concentration as denoted). (B) Experimental and simulated steam generation rates as a function of concentration in nanoparticle solutions of nanoshells (red) and nanomatryoshkas (blue) illuminated at laser intensities of $29 \mathrm{~W} / \mathrm{cm}^{2}$ (diamonds), $42 \mathrm{~W} / \mathrm{cm}^{2}$ (circles), and $57 \mathrm{~W} / \mathrm{cm}^{2}$ (squares). Dotted lines are a fit through the first three data points as a guide to the eye and to illustrate deviation from linear behavior. 
Another important characteristic of light-induced steam generation is the steady state steam generation rate, defined as the slope of the mass loss versus time once a linearly increasing mass loss is achieved (Figure 3B). This is investigated experimentally by varying both the particle concentration and the illumination intensity. For nanoshells, the steam generation rate saturates at nominally $0.9 \times 10^{10} \mathrm{NP} / \mathrm{ml}$, which is the same concentration where the fractional light absorption saturates in our calculations. For nanomatryoshkas, there is no clear saturation concentration within our experimental range, which is also in agreement with our theoretical description. Comparing the two types of nanoparticles, we observe that the steam generation rates for nanoshell solutions are almost a factor of 2 larger than for nanomatryoshka solutions of equal concentration and illumination intensity. This is also a direct yet unexpected result of the larger maximum heat source densities in nanoshell solutions caused by light trapping since the two particles have nearly equal absorption cross sections. The effects realized here in the localization of heat will only persist when the heat source dimensions are larger than the heat diffusion length of the solvent. Theoretically, a quantity proportional to the steam generation rate can be calculated. The energy per unit time $P_{\mathrm{CC}}$ is transferred across a convective cooling boundary at the solution/air interface. Dividing this quantity by the enthalpy of vaporization of water yields a value proportional to the experimental steam generation rate (Figure 3B). This simple energy loss model illustrates that classical heat transfer with the light trapping-derived heat source density as input reproduces the trends seen in the experiment.

We also examine the consequences of collective thermal effects in an ensemble of illuminated particles for concentrations within the multiple scattering regime. In this regime, the interparticle spacing is on the order of $10 \mu \mathrm{m}$ and the thermal diffusivity of water gives times on the order of 10-100 $\mu$ s for heat to diffuse from a nanoparticle to its nearest neighbor. For shorter time scales, the particles heat in an isolated and independent manner; for longer times, collective effects, that is, the heating of a fluid due to multiple nanoparticles, must be considered. We examine collective heating effects by the illuminated particles by approximating the solution of randomly positioned nanoparticles as a three-dimensional lattice (Figure 4A) with constant $a$, determined by the particle concentration. If the width of the Gaussian laser beam, $w$, and the decay length of the light in the medium, $d$, are sufficiently large such that $a \leqslant w$ and $a \leqslant d$, then each particle absorbs the same amount of power as its immediate neighbors. In this case, the heat fluxes into and out of an idealized unit cell of the lattice in Figure 4A cancel completely. This results in zero net heat flux and allows for simple calculations of the time-dependent temperature increase and the resulting vaporization of the surrounding solvent $\left(\mathrm{H}_{2} \mathrm{O}\right)$. An experiment to observe the onset of steam production was performed with temporal and mass resolution sufficient to observe both background evaporation and steam production. Statistical analysis of the background evaporation rate provided a means of calculating the earliest onset of steam production (Figure 4B). These values lie well within the range predicted from the simple lattice model (Figure 4B, shaded red area), using both the time to reach the boiling point of water as a lower bound (dashed line) and the time to fully vaporize the unit cell volume (solid line) as an upper bound. These data reveal a mechanism for steam generation that is consistent with the Fourier law of heat conduction. 
A

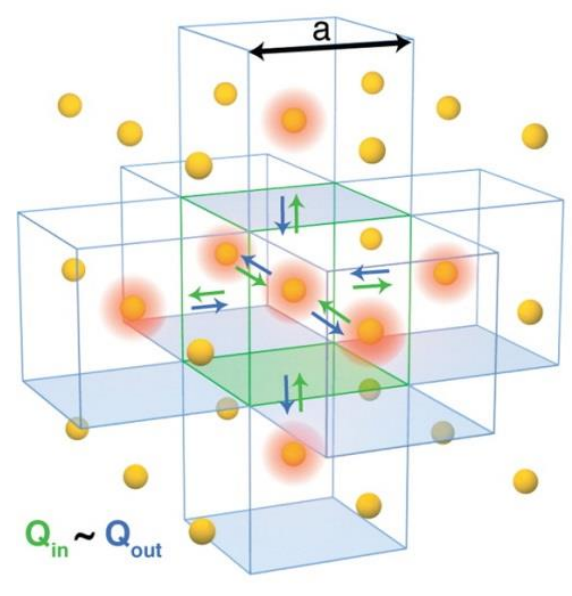

B

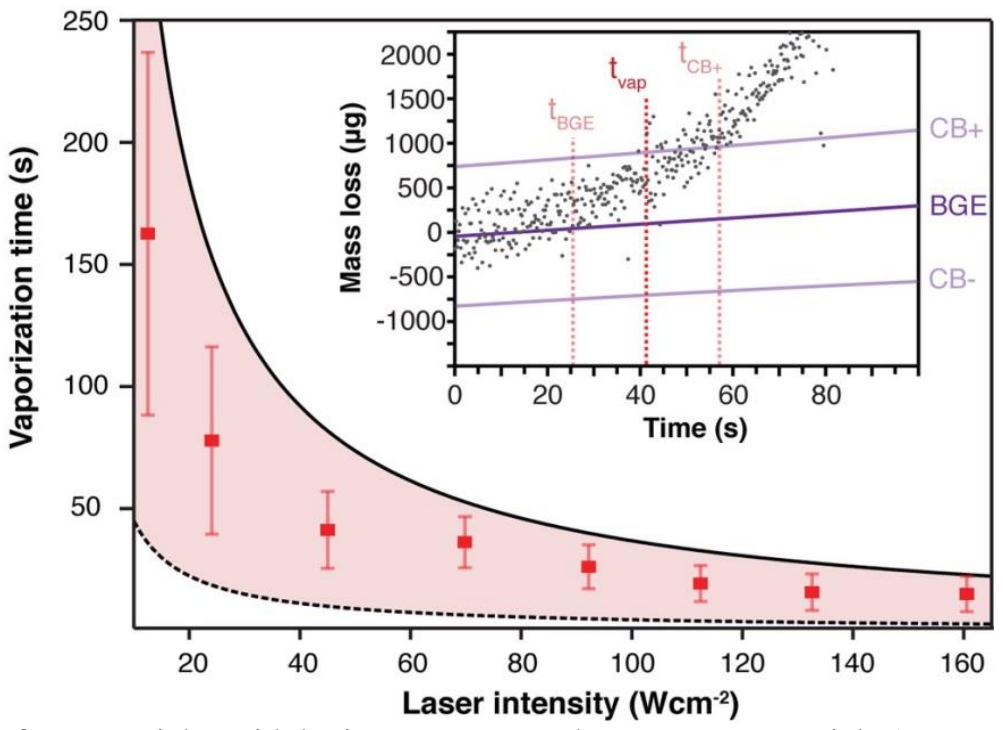

Figure 4. (A) Schematic illustrating a lattice of nanoparticles with lattice constant $a$. The center nanoparticle (green bounding box) in the lattice emits the same amount of heat, $Q_{\text {out }}$ (green arrows), as its nearest neighbors (blue bounding boxes), which emit heat $Q_{\text {in }}$ (blue arrows). (B) Vaporization times measured experimentally for 8 laser intensities (red symbols), contrasted with that predicted from the lattice model in panel A denoted by the red shaded region. Inset: Method for experimentally obtaining vaporization time. Background evaporation (BGE) is found, with $95 \%$ confidence bands $(\mathrm{CB}+$ and $\mathrm{CB}-)$. $t_{\text {vap }}$ is the average of the $t_{\mathrm{BGE}}$ and $t_{\mathrm{CB}+}$, the time at which mass loss statistically rises above $\mathrm{BGE}$ and $\mathrm{CB}+. t_{\mathrm{BGE}}$ and $t_{\mathrm{CB}+}$ also define the error for $t_{\mathrm{vap}}$.

The optical properties of nanoparticle ensembles underlie many of the emerging applications of plasmonics, from photothermal cancer therapies to energy harvesting. This study clearly indicates that both the scattering and absorption characteristics of nanoparticles, in addition to particle concentration, need to be critically considered in our understanding of particle-based photothermal processes. Our finding that light trapping through multiple scattering can concentrate energy absorption into very small volumes is a crucially important insight that paves the way for further optimization and development of photothermal applications such as light-induced steam production.

\section{Materials and Methods}

\section{Nanoparticle Synthesis}

The synthesis of gold nanoshells followed standard methods detailed in previous work. $(34,35)$ A detailed explanation of the synthesis of the gold nanomatryoshkas can also be found in recent literature. $(28,29)$

\section{Nanoparticle Characterization}

Nanoparticle cross sections were calculated by comparing Mie theory calculations with UV-vis extinction spectra. The particle dimensions (core and shell radii) were tuned in the Mie theory calculations until the extinction spectrum matched that obtained from the UV-vis. Overall nanoparticle diameters were confirmed with a scanning tunneling microscope (see Figure S2, Supporting Information). We determined the nanoparticle concentration of our solutions by 
measuring the extinction spectrum of a very dilute solution $\left(<10^{7-8} \mathrm{NP} / \mathrm{ml}\right)$ and utilizing the formula:

$$
N=\frac{2.303 A}{\pi r^{2} Q_{e x t} d}
$$

where $N$ is the nanoparticle concentration, $A$ is the absorbance at the resonance wavelength, $r$ is the radius of the nanoparticle, $Q_{\text {ext }}$ is the extinction efficiency, and $d$ is the path length of the light in the solution.

\section{Experimental Methods}

Nanoparticle solution $(3.5 \mathrm{~mL})$ in cuvettes with dimensions $1 \times 1 \times 4 \mathrm{~cm}$ were placed on a scale (Torbal, AD500) with mass resolution of $1 \mathrm{mg}$. The solutions were illuminated by an 808 $\mathrm{nm}$ CW fiber laser (Diomed, 15Plus, Angio Dynamics) with a spot size of $3 \mathrm{~mm}$ incident from the top of the solution. The mass was recorded every $5 \mathrm{~s}$. The laser output power was tunable between 0.5 and $15 \mathrm{~W}$. Two infrared thermocouples (Omega OS-801-MT-K) with a $0.5 \mathrm{~cm}$ spot size measured the temperature of the top and bottom of the solution every $5 \mathrm{~s}$. See Figure S3, Supporting Information for a schematic of the experimental setup. Data obtained for Figure 4 required the use of a scale (Sartorius) with a time resolution of $50 \mu \mathrm{s}$. The same Diomed laser was utilized for these experiments incident, again, from the top of the solution. We utilized primarily scattering nanoparticles (silica, $80 \mathrm{~nm}$ diameter) in solution as a control experiment to determine the effect of light absorption in the water of the illuminated system. With dense silica NP solutions of equal optical density with each concentration of nanoshell and nanomatryoshka solution, the steam production rate was not measurable above the background evaporation rate.

\section{Theoretical Methods}

The Monte Carlo simulations were custom written in $C$, and tested against common analytic solutions for simple geometries and parameters (see Supporting Information). The heat transfer calculations were performed using the finite-element method (FEM, Comsol). The experimental geometry of the system was modeled as closely as possible including cuvette size and material, the aluminum resting plate of the scale, and the surrounding environment (see Figure S3, Supporting Information, for a schematic). 


\section{Supporting Information}

Discussion of the methods used for simulating radiative transport; scanning electron micrographs of the nanoparticles and Mie theory cross sections for the same; schematic of the experimental setup and simulation geometry utilized; photographs of nanoparticle solutions in cuvettes. This material is available free of charge via the Internet at http://pubs.acs.org.

\# Author Contributions

(N.J.H. and A.S.U.) These authors contributed equally to this work.

The authors declare no competing financial interest.

\section{Acknowledgment}

The authors would like to acknowledge Mark Knight, Oara Neumann, and Surbhi Lal for productive discussions. This work was supported by the Bill and Melinda Gates Foundation, the Robert A. Welch Foundation under Grants C-1220 (to N.J.H.) and C-1222 (to P.N.), the National Security Science and Engineering Faculty Fellowship (NSSEFF) N00244-09-1-0067, and the AFOSR MURI Program. 


\section{References}

1. Service, R. F.Turning up the Light Science 2013, 342, 794- 795

2. Currie, M. J.; Mapel, J. K.; Heidel, T. D.; Goffri, S.; Baldo, M. A.High-Efficiency Organic Solar Concentrators for Photovoltaics Science 2008, 321, 226- 228

3. Gabor, N. M.; Song, J. C. W.; Ma, Q.; Nair, N. L.; Taychatanapat, T.; Watanabe, K.; Taniguchi, T.; Levitov, L. S.; Jarillo-Herrero, P.Hot Carrier-Assisted Intrinsic Photoresponse in Graphene Science 2011, 334, 648- 652

4. Neumann, O.; Urban, A. S.; Day, J.; Lal, S.; Nordlander, P.; Halas, N. J.Solar Vapor Generation Enabled by Nanoparticles ACS Nano 2013, 7, 42- 49

5. Lukianova-Hleb, E.; Hu, Y.; Latterini, L.; Tarpani, L.; Lee, S.; Drezek, R. A.; Hafner, J. H.; Lapotko, D. O.Plasmonic Nanobubbles as Transient Vapor Nanobubbles Generated around Plasmonic Nanoparticles ACS Nano 2010, 4, 2109- 2123

6. Boriskina, S. V.; Ghasemi, H.; Chen, G.Plasmonic Materials for Energy: From Physics to Applications Mater. Today 2013, 16, 375- 386

7. Govorov, A. O.; Zhang, W.; Skeini, T.; Richardson, H.; Lee, J.; Kotov, N. A.Gold Nanoparticle Ensembles as Heaters and Actuators: Melting and Collective Plasmon Resonances Nanoscale Res. Lett. 2006, 1, 84- 90

8. Baral, S.; Green, A. J.; Livshits, M. Y.; Govorov, A. O.; Richardson, H. H.Comparison of Vapor Formation of Water at the Solid/Water Interface to Colloidal Solutions Using Optically Excited Gold Nanostructures ACS Nano 2014, 8, 1439- 1448

9. Govorov, A. O.; Richardson, H. H.Generating Heat with Metal Nanoparticles Nano Today 2007, 2, 30- 38

10. Richardson, H. H.; Carlson, M. T.; Tandler, P. J.; Hernandez, P.; Govorov, A. O.Experimental and Theoretical Studies of Light-to-Heat Conversion and Collective Heating Effects in Metal Nanoparticle Solutions Nano Lett. 2009, 9, 1139- 1146

11. Baffou, G.; Berto, P.; Bermúdez Ureña, E.; Quidant, R.; Monneret, S.; Polleux, R. J.; Rigneault, H.Photoinduced Heating of Nanoparticle ACS Nano 2013, 7, 6478-6488

12. Chabanov, A.; Stoytchev, M.; Genack, A.Statistical Signatures of Photon Localization Nature 2000, 404, 850- 853

13. Wolf, P.; Maret, G.Weak Localization and Coherent Backscattering of Photons in Disordered Media Phys. Rev. Lett. 1985, 55, 2696- 2699

14. Van Albada, M. P.; Lagendijk, A.Observation of Weak Localization of Light in a Random Medium Phys. Rev. Lett. 1985, 55, 2692- 2695

15. Segev, M.; Silberberg, Y.; Christodoulides, D. N.Anderson Localization of Light Nat. Photonics 2013, 7, 197-204

16. Wiersma, D. S.; Bartolini, P.; Lagendijk, A.; Righini, R.Localization of Light in a Disordered Medium Nature 1997, 390, 671-673

17. Ishimaru, A. Wave Propagation and Scattering in Random Media; IEEE Press: Piscataway, NJ, 1978.

18. Chandrasekhar, R. Radiative Transfer; Dover Publications, Inc.: New York, 1960.

19. Muskens, O. L.; Rivas, J. G.; Algra, R. E.; Bakkers, E. P. A. M.; Lagendijk, A.Design of Light Scattering in Nanowire Materials for Photovoltaic Applications Nano Lett. 2008, 8, $2638-2642$

20. Tjahjono, I. K.; Bayazitoglu, Y.Near-Infrared Light Heating of a Slab by Embedded Nanoparticles Int. J. Heat Mass Transfer 2008, 51, 1505- 1515 
21. Vera, J.; Bayazitoglu, Y.Gold Nanoshell Density Variation with Laser Power for Induced Hyperthermia Int. J. Heat Mass Transfer 2009, 52, 564- 573

22. Qin, Z.; Bischof, J. C.Thermophysical and Biological Responses of Gold Nanoparticle Laser Heating Chem. Soc. Rev. 2012, 41, 1191

23. Elliott, A. M.; Stafford, R. J.; Schwartz, J.; Wang, J.; Shetty, A. M.; Bourgoyne, C.; O’Neal, P.; Hazle, J. D.Laser-Induced Thermal Response and Characterization of Nanoparticles for Cancer Treatment Using Magnetic Resonance Thermal Imaging Med. Phys. 2007, 34, 3102

24. Roper, D. K.; Ahn, W.; Hoepfner, M.Microscale Heat Transfer Transduced by Surface Plasmon Resonant Gold Nanoparticles J. Phys. Chem. C 2007, 111, 3636-3641

25. Ramella-Roman, J. C.; Prahl, S. A.; Jacques, S. L.Three Monte Carlo Programs of Polarized Light Transport into Scattering Media: Part I Opt. Express 2005, 13, 4420

26. Yang, W.-J.; Taniguchi, H.; Kudo, K. Advances in Heat Transfer: Radiative Heat Transfer by the Monte Carlo Method; Academic Press: San Diego, CA, 1995; Vol. 1995.

27. Prodan, E.; Radloff, C.; Halas, N. J.; Nordlander, P.A Hybridization Model for the Plasmon Response of Complex Nanostructures Science 2003, 302, 419-422

28. Ayala-Orozco, C.; Liu, J. G.; Knight, M. W.; Wang, Y.; Day, J. K.; Nordlander, P.; Halas, N. J.Fluorescence Enhancement of Molecules inside a Gold Nanomatryoshka Nano Lett. 2014, 14, 2926- 2933

29. Ayala-Orozco, C.; Urban, C.; Knight, M. W.; Urban, A. S.; Neumann, O.; Bishnoi, S. W.; Mukherjee, S.; Goodman, A. M.; Charron, H.; Mitchell, T.Au Nanomatryoshkas as Efficient Transducers for Cancer Treatment: Benchmarking against Nanoshells ACS Nano 2014, 8, 6372-6381

30. Hofmann, C. E.; de Abajo, F. J. G.; Atwater, H. A.Enhancing the Radiative Rate in III-V Semiconductor Plasmonic Core-Shell Nanowire Resonators Nano Lett. 2011, 11, 372376

31. Muskens, O. L.; Giannini, V.; Sánchez-Gil, J. A.; Gómez Rivas, J.Optical Scattering Resonances of Single and Coupled Dimer Plasmonic Nanoantennas Opt. Express 2007, 15,17736

32. Coenen, T.; Bernal Arango, F.; Femius Koenderink, A.; Polman, A.Directional Emission from a Single Plasmonic Scatterer Nat. Commun. 2014, 5, 3250

33. Tam, W. G.; Zardecki, A.Multiple Scattering Corrections to the Beer-Lambert Law. 1: Open Detector Appl. Opt. 1982, 21, 2405-2412

34. Oldenburg, S. J.; Averitt, R. D.; Westcott, S. L.; Halas, N. J.Nanoengineering of Optical Resonances Chem. Phys. Lett. 1998, 288, 243- 247

35. Brinson, B. E.; Lassiter, J. B.; Levin, C. S.; Bardhan, R.; Mirin, N.; Halas, N. J.Nanoshells Made Easy: Improving Au Layer Growth on Nanoparticle Surfaces Langmuir 2008, 24, 14166-14171 